\title{
Susceptibility to infection due to TYK2 deficiency
}

INSERM

\section{Source}

INSERM. (1999). Orphanet: an online rare disease and orphan drug data base.

Susceptibility to infection due to TYK2 deficiency. ORPHA:331226

Susceptibility to infection due to TYK2 deficiency is a rare primary immunodeficiency characterized by increased susceptibility to intracellular bacterial and viral infection, with or without increased serum IgE. Clinical manifestations are highly variable, depending on the infection type and location, and can include recurrent otitis, sinusitis, pulmonary and cutaneous infections, meningitis and internal abscesses. 